\title{
DE BARBADOS A SAMOA: REPASO A LOS PRINCIPALES HITOS PARA LOS INTERESES DE LOS PEQUEÑOS ESTADOS INSULARES EN DESARROLLO DESDE 1994 HASTA 2014
}

\author{
Mario J. Gallego ${ }^{1}$ \\ Universidad Nacional de Educación a Distancia (UNED)
}

\begin{abstract}
Resumen:
Los pequeños Estados insulares en desarrollo precisaban de una voz común que se pudo escuchar por primera vez en Barbados, en 1994, durante la primera Conferencia celebrada para tratar los asuntos propios de estos espacios vulnerables por su tamaño y condición insular. El despegue posterior de este grupo de Estados en la arena internacional hasta la recientemente celebrada tercera Conferencia, en septiembre de 2014 en Samoa, es el tema central del artículo. El repaso a los diferentes momentos clave sucedidos durante estas dos décadas permite constatar los asuntos prioritarios para este grupo de países en cada contexto y sus avances hacia la completa institucionalización de sus asuntos por parte de la Organización de las Naciones Unidas.
\end{abstract}

Palabras clave: SIDS, Estados insulares en desarrollo, islas, ONU, SIDS, UN-OHRLLS.

Title in English: "From Barbados to Samoa: Reviewing the main Landmarks for Small Island Developing States from 1994 to 2014"

\begin{abstract}
:
Small island developing States were long time needing a common voice to address their specific needs. This voice could be heard worldwide for the first time in Barbados, in 1994, during the first Conference held to discuss the specific affairs that are important for these geographical areas that are vulnerable because of their size and insular condition. The subsequent progress of this group of States in the international arena during two decades until the third Conference on held recently in September 2014 in Samoa, is the main subject of this essay. Reviewing the key moments happened between these two international events allows to note the priority issues considered by this group of countries in each context and their progress towards full institutionalization of their affairs by the United Nations.
\end{abstract}

Keywords: SIDS, small island developing states, islands, UN, UN-OHRLLS.

Copyright (C) UNISCI, 2015.

Las opiniones expresadas en estos artículos son propias de sus autores, y no reflejan necesariamente la opinión de UNISCI. The views expressed in these articles are those of the authors, and do not necessarily reflect the views of UNISCI.

\footnotetext{
${ }^{1}$ Mario J. Gallego Cosme es consultor del PNUD en República Dominicana y doctorando en seguridad internacional en el Instituto Universitario "General Gutiérrez Mellado" de Madrid.

E-mail: mgallego136@alumno.uned.es.
} 


\section{Introducción}

La visibilidad de las problemáticas comunes a las islas pequeñas, sean estas Estados, parte de Estados continentales o dependencias, en buena medida ha ido de la mano de los asuntos medioambientales, pues los efectos del llamado cambio climático son los que mayor preocupación suscitan en estos espacios geográficos de alta vulnerabilidad. Sin embargo, los intereses comunes de los 38 países que la Organización de las Naciones Unidas considera pequeños Estados insulares en desarrollo (SIDS en adelante, por sus siglas en inglés) debido a sus especiales circunstancias derivadas del tamaño y la insularidad, han podido ir cuajando en la agenda internacional como un asunto de vital importancia para ser tratado por medio de foros concretos. En este sentido, si bien los primeros pasos han sido tardíos, pues la primera gran iniciativa focalizada en estos Estados, como se verá en este trabajo, data de 1994, también es cierto que desde ese momento se ha avanzado mucho. Este hecho en parte se explica por la atención prestada por la Comunidad Internacional a asuntos concretos que permiten canalizar las necesidades propias de los SIDS (como es el caso de los Objetivos del Milenio o los temas vinculados al medioambiente), aunque también es debido a la confluencia de intereses comunes entre los Estados insulares y los de algunos países ribereños a los que también preocupan aspectos concretos propios de islas pequeñas, como es el caso de territorios continentales situados a escasa altura sobre el nivel del mar.

En las siguientes páginas se realizará un repaso cronológico a través de los diferentes momentos históricos que representaron un avance para el grupo de los SIDS en la arena internacional. Todos estos hitos se llevaron a cabo bajo los auspicios de las Naciones Unidas, aunque bajo dos formas diferenciadas: a través de Cumbres internacionales que tangencialmente incluyeron a los SIDS (todas ellas sobre medioambiente) y por medio de las tres Conferencias especialmente celebradas para tratar en exclusiva los temas que atañen al desarrollo de los SIDS y sus poblaciones. El primero de estos eventos fue la Cumbre de Río en 1992, que es el primer antecedente de la institucionalización definitiva de las Conferencias sobre SIDS que empezarían a celebrarse cada década desde su primera edición en Barbados en 1994. Seguidamente se examina la revisión que se realiza en la $22^{a}$ sesión extraordinaria de la Asamblea General de la ONU de 1999 acerca de lo acordado cinco años antes en el país caribeño y la subsiguiente creación de la primera oficina especializada en Estados insulares en desarrollo, ya en 2001. Esta secuencia temporal en la que se celebraron los mencionados eventos de la década de los '90 — Cumbre de la Tierra, Conferencia sobre SIDS y revisión quinquenal de esta - se repite nuevamente en los primeros años del siglo XXI con la Cumbre de Johannesburgo de 2002, la Conferencia de los SIDS de Mauricio en 2005 y la revisión de esta en 2010. Finalmente, la nueva Cumbre de Río de Janeiro de 2012 abre un nuevo ciclo cuyo siguiente paso ha sido la tercera - y última hasta la fecha- Conferencia de SIDS, celebrada en septiembre de 2014 en Samoa.

Cada uno de estos eventos será revisado en virtud de sus aportes principales a través de sus más importantes documentos, por lo que las referencias a los textos y resoluciones de las Naciones Unidas serán recurrentes. Con este repaso se podrá comprobar la situación actual en la que institucionalmente se encuentra este grupo de países con necesidades especiales. Al respecto se puede adelantar el hecho de que en cada evento internacional se llamó la atención sobre los esfuerzos que debían seguir realizándose (y que aún restan por concretar en la actualidad), tanto en materia medioambiental como en los diversos aspectos que fueron trasladándose hacia las prioridades que debían ser tratadas. En este sentido se hace evidente que el estudio de las problemáticas que preocupan a los SIDS pasa por un acercamiento interdisciplinar que toque aspectos que van más allá de los vinculados con el medio natural y que deben considerar las vinculaciones de las sociedades de estos espacios con sus propios 
gobiernos y con el ámbito internacional en los más diversos órdenes de lo cultural, lo económico, la planificación territorial, la tecnología, la educación o la salud.

\section{Conferencia de las Naciones Unidas sobre el Medioambiente y el Desarrollo de 1992}

Este importante evento, también conocido como la Cumbre de la Tierra de Río de Janeiro de 1992 (UNCED por sus siglas en inglés), fue el antecedente inmediato de la que sería la primera Conferencia sobre islas pequeñas en desarrollo. En esta ciudad brasileña es donde por primera vez se abordaron oficialmente los problemas a los que los SIDS deben hacer frente de manera pormenorizada. Esta cumbre, celebrada entre el 2 y el 14 de junio de 1992, es conocida por la enorme cantidad de documentación que produjo y por su influencia posterior a nivel internacional, ya que comúnmente se entiende que es el antecedente inmediato a la firma del Protocolo de Kyoto de 1997. En efecto, de la misma emanan tres documentos de gran relevancia: la Declaración de Río sobre Medioambiente y Desarrollo ${ }^{2}$, la ["non-binding authoritative"] Declaración de Principios para el Consenso Global sobre la Gestión, Conservación y Desarrollo Sostenible de todos los tipos de Bosques ${ }^{3}$, y la Agenda $21^{4}$.

En este último texto, específicamente de su capítulo 17, que versa sobre la "protección de los océanos, todos los tipos de mares, incluyendo los enclaustrados y semi-enclaustrados, y áreas costeras y la protección, uso racional y desarrollo de sus recursos vivos", se menciona a los SIDS y a las zonas litorales por su vulnerabilidad ${ }^{5}$. En estos párrafos se habla del alto valor de estos espacios en términos de biodiversidad - debido al aislamiento, que es una cuestión que incide en otros aspectos como el tamaño-, de sus recursos limitados, y de los problemas a los que las islas pequeñas deben hacer frente debido a su condición geográfica. A continuación, la Agenda 21 (1992: 17.128) enumera de manera exhaustiva y pormenorizada las actividades a cumplir en relación a los SIDS, de entre las que destaca el "estudio de las características ambientales y de desarrollo especiales de las islas pequeñas", la preparación de planes para la utilización inteligente de los recursos, la ordenación territorial de las zonas costeras, la participación comunitaria en la planificación, la revisión de las políticas actuales y el fomento de la coordinación intersectorial ${ }^{6}$.

Para llevar a cabo dichas recomendaciones y para dar seguimiento a las condiciones de vulnerabilidad de los pequeños Estados insulares en desarrollo, se insta a la celebración de la que iba a ser la primera conferencia sobre esta temática, la cual debía celebrarse al año siguiente, en 1993 bajo la premisa de que resulta necesaria la cooperación internacional para abordar las problemáticas comunes a todos los espacios geográficos de este tipo en el nivel planetario $^{7}$. En este sentido, Cumbre de la Tierra de Río de Janeiro, y más concretamente su principal documento, la extensa Agenda 21, se enarbola como el principal instrumento

\footnotetext{
2 "Rio Declaration on environment and development", Annex I. A/CONF.151/26 vol. I, Report of the United Nations conference on environment and development, Rio de Janeiro (3-14 de junio de 1992), en http://www.un.org/documents/ga/conf151/aconf15126-1annex1.htm.

3 "Non legally binding authoritative statement of principles for a global consensus on the management, conservation and sustainable development of all types of forests", Annex III. A/CONF.151/26 vol. III, Report of the United Nations conference on environment and development, Rio de Janeiro (3-14 de junio de 1992), en http://www.un.org/documents/ga/conf151/aconf15126-3annex3.htm.

4 "Agenda 21", Annex II. A/CONF.151/26 vol. II, Report of the United Nations conference on environment and development, Rio de Janeiro (3-14 de junio de 1992), en http://sustainabledevelopment.un.org/content/documents/Agenda21.pdf.

${ }^{5}$ Ibid., c. 17. arts.123-6.

${ }^{6}$ Ibid., c. 17 art. 128.

${ }^{7}$ Ibid., c. 17 . art. 131.
} 
valedor de la que sería la primera Conferencia sobre SIDS, que se celebraría en Barbados en 1994, y que será revisada a continuación.

\section{Conferencia Global de las Naciones Unidas sobre el Desarrollo Sostenible de los Pequeños Estados Insulares en Desarrollo de 1994}

La Asamblea General de la ONU, haciéndose eco de la recomendación de la Agenda 21, solicita en diciembre de 1992, durante la $93^{\circ}$ sesión plenaria de ese año, la celebración de la conferencia para abril de $1994^{8}$. Así, Bridgetown fue la sede, desde el 26 de abril hasta el 6 de mayo de ese año, del primer evento mundial específicamente destinado a los SIDS y del cual se extrae el documento que, hasta la fecha, es el principal referente internacionalmente aprobado en la materia; el Programa de Acción para el desarrollo sostenible de los pequeños Estados insulares en desarrollo ${ }^{9}$, que va anexo al Reporte de la Conferencia Global de las Naciones Unidas para el Desarrollo Sostenible de los Pequeños Estados Insulares en Desarrollo ${ }^{10}$.

Este texto, también conocido como Plan de Acción de Barbados (Barbados Plan of Action, o BPOA), tiene dos partes contrastadas: una en la que elabora las vulnerabilidades a las que las islas pequeñas deben hacer frente, y la otra en la que se comentan las estrategias encaminadas a reducir dichas vulnerabilidades (en el último capítulo: "aplicación, vigilancia y examen"). Para su puesta en funcionamiento se delega la responsabilidad a la Comisión para el Desarrollo Sostenible, que había sido creada a final de 1992 para dar seguimiento a lo acordado en la Cumbre de Río. Como se explica en el punto 15 del BPOA, ese documento se concentra en "las medidas inmediatas que pueden emprenderse en el proceso general hacia el desarrollo sostenible", ya que en ese mismo apartado se recuerda que "el desarrollo sostenible es un proceso, y no un fenómeno". Estas medidas se recogen agrupadas en torno a tres niveles de actuación (nacional, regional e internacional) en las "14 esferas prioritarias" que se listan a continuación ${ }^{11}$ :

- Cambios climáticos y aumento del nivel del mar

- Desastres naturales y ambientales

- Gestión de desechos

- Recursos costeros y marinos

- Recursos de agua dulce

- $\quad$ Recursos de tierras

- Recursos energéticos

- Recursos turísticos

- Recursos relativos a la diversidad biológica

\footnotetext{
8 "Convening of a global conference on the sustainable development of small island developing States", UN General Assembly, $47^{\text {th }}$ session, resolution adopted on 22 December 1992, A/RES/47/189, en http://www.un.org/documents/ga/res/47/a47r189.htm.

9 "Programme of Action for the Sustainable Development of Small Island Developing States", Annex II. A/CONF.167/9, Report of the Global Conference on the Sustainable Development of Small Island Developing States, Bridgetown, Barbados (26 April - 6 May 1994), en http://www.un.org/ga/search/view_doc.asp?symbol=A/CONF.167/9.

${ }^{10}$ Ibid.

${ }^{11}$ Ibid., Annex II, art. 16.
} 
- Instituciones nacionales y capacidad administrativa

- Instituciones regionales y cooperación técnica

- $\quad$ Transporte y comunicaciones

- Ciencia y tecnología

- Perfeccionamiento de los recursos humanos

Tal y como se hiciera en la Agenda 21, aspectos como la asolación y el tamaño fueron tomados en consideración, así como otros relativos a las altas densidades poblacionales y su efecto en la presión por los recursos limitados, la excesiva dependencia en el comercio internacional y el efecto multiplicador de la vulnerabilidad que pueden presentar los Estados $\operatorname{archipielágicos}^{12}$. Todas estas cuestiones condicionantes serán tópicos que se repetirán en numerosas ocasiones en los diversos documentos que se elaboran en los años posteriores. Finalmente, el BPOA identifica áreas sectoriales de actuación como son la capacitación, el desarrollo institucional a todos los niveles, la cooperación en la transferencia de tecnologías amigables con el medioambiente, y los asuntos relacionados con las finanzas, la diversificación de la economía y el comercio. Además del BPOA, un segundo documento llamado "Declaración de Barbados" también se encuentra incluido en el reporte ${ }^{13}$ en el que se explica y justifica el Plan de Acción. Destaca de esta Declaración el papel de "los recursos humanos y el patrimonio cultural"14 en el desarrollo sostenible, la responsabilidad que tiene la comunidad internacional en conjunto ${ }^{15}$ y el hecho de que los SIDS se vean afectados como ningún otro Estado de los efectos del conocido Cambio Climático al que otros han contribuido en mayor grado ${ }^{16}$.

\section{Sesión Especial 22 de la Asamblea General de las Naciones Unidas de 1999}

El motivo de esta sesión extraordinaria hay que buscarlo en la cuarta sesión de trabajo de la Comisión para el Desarrollo Sostenible de abril-mayo de $1996^{17}$, de donde se desprende en su $16^{\text {a }}$ decisión que es necesario trazar la hoja de ruta que pasa por convocar en 1997 una sesión extraordinaria de la Asamblea General en la que se extraerían los puntos a tratar en una revisión más en profundidad del BPOA que se llevaría a cabo ya en 1999. De esta manera, durante la sesión especial número 19 de la Asamblea General de la ONU de junio de 1997, conocida como "Earth Summit +5 ", se realiza una comprobación de las áreas que necesitaban mayor impulso de la Agenda 21 con motivo del quinto aniversario de su elaboración y se insta a que se organice la mencionada revisión del Barbados Plan of Action a los dos años desde esa fecha ${ }^{18}$.

\footnotetext{
${ }^{12}$ Ibid., Resol. 1, Annex II, art. 4.

${ }^{13}$ Ibid., Resol. 1, Annex I.

${ }^{14}$ Ibid., Resol. 1, Annex I, part 1, I.1.

${ }^{15}$ Ibid., Resol. 1, Annex I, part 2, I.1.

${ }^{16}$ Ibid., Resol. 1, Annex I, part 1, III.2.

${ }^{17}$ ECOSOC, UN Commission on Sustainable Development, Report on the $4^{\text {th }}$ session, E/CN.17/1996/38. (18 de abril - 3 de mayo de 1996), en: http://www.un.org/ga/search/view_doc.asp?symbol=E/CN.17/1996/38.

${ }^{18}$ UN General Assembly, $19^{\text {th }}$ special session, Resolutions and decisions adopted from June 23 to 28, 1997, sec. III, III. A/S 19/33, en

http://www.un.org/en/ga/search/view_doc.asp?symbol=A/S-19/33.
} 
La Sesión Especial 22 de la Asamblea General de las Naciones Unidas del 27 y 28 de septiembre de 1999 fue bautizada como "BPOA+5" por tratar en exclusiva el progreso del Programa de Acción para el desarrollo sostenible de los pequeños Estados insulares en desarrollo que había sido firmado en Barbados cinco años antes ${ }^{19}$. En la parte declarativa del texto, acerca del proceso para la puesta en marcha del BPOA, que contiene un reconocimiento al papel de los SIDS como "guardianes de vastas áreas oceánicas del mundo" no recogido con anterioridad en ningún otro documento oficial, insta a incrementar los esfuerzos de todas las partes involucradas y de la comunidad internacional ${ }^{20}$.

En la parte evaluativa del documento se pone de manifiesto que, sin menosprecio del resto de áreas sectoriales, se identifican seis de especial importancia prioritaria, que se citan a continuación en el orden en que se exponen en el texto ${ }^{21}$ : cambio climático y aumento del nivel del mar, desastres naturales, recursos de agua dulce, recursos costeros y marinos, energía y turismo. Además de los consejos concretos que se repasan para cada uno de esos puntos críticos, el reporte recoge una sección de implementación, que elabora los siguientes puntos $^{22}$ : estrategias de desarrollo sostenible, capacitación, cooperación, liberalización y globalización [del comercio], la transferencia de tecnologías amigables con el medioambiente, la consecución de un índice de vulnerabilidad y la creación de una red de información entre SIDS. Estos dos últimos puntos, además del énfasis en el libre comercio, son la principal novedad respecto al BPOA y a la Agenda 21, aunque de alguna manera recogen lo que se había trabajado en la sexta sesión de trabajo de la Comisión para el Desarrollo Sostenible del 22 de diciembre de 1997 y del 20 de abril al 1 de mayo de $1998^{23}$.

\section{Creación de la "Oficina del Alto Representante de las Naciones Unidas para los países menos desarrollados, Estados enclavados en desarrollo y los pequeños Estados insulares en desarrollo", en 2001}

Durante la Tercera Conferencia sobre los Países Menos Desarrollados de Bruselas de mayo del 2001 se pone de manifiesto la importancia de dar seguimiento oficial a esta este grupo de Estados en situación de desventaja —aunque en la parte declarativa no se mencionan las islas- y se pide formalmente al Secretario General que se asegure de ello ${ }^{24}$. Es necesario comentar que en esta Conferencia sobre los Países Menos Desarrollados se vuelve a tratar el tema de las islas respecto al desarrollo ${ }^{25}$, ya que, al menos desde la Segunda Conferencia de este tipo, la celebrada en París en 1990, es comentada esta preocupación por la situación de "los países insulares" (sic) ${ }^{26}$.

\footnotetext{
${ }^{19}$ UN General Assembly, $22^{\text {nd }}$ special session, Resolutions and decisions adopted on September 27 and $28,1999$. A/S 22/11, en http://www.un.org/en/ga/search/view_doc.asp?symbol=A/S-22/11(SUPP.

${ }^{20}$ Ibid., Decl, par. 8.

${ }^{21}$ Ibid., III, I, 1.

${ }^{22}$ Ibid., III, III.

${ }^{23}$ ECOSOC, UN Commission on Sustainable Development, Report on the $6^{\text {th }}$ session $(22$ de diciembre de 1997 y 20 de abril - 1 de mayo 1998). E/CN.17/1998/20, en http://www.un.org/ga/search/view doc.asp?symbol=E/CN.17/1998/20.

24 "Report of the third United Nations conference on the Least Developed Countries", Brussels (14-20 de mayo 2001), sec. I, 10 y II, 96-8. A/CONF.191/12, en http://www.un.org/en/conf/lde/pdf/aconf191d13.en.pdf.

${ }^{25}$ Ibid., II, 8 .

${ }^{26}$ Report of the second United Nations conference on the Least Developed Countries, Paris (3-14 de septiembre de 1990), arts. 125-7. A/CONF.147/18, en

http://www.un.org/en/conf/ldc/pdf/outcome_\%20document_second_un_conference_\%201dcs.\%20pdf.pdf.
} 
Ya en diciembre del 2001, en vista de la necesidad aludida y de las demandas realizadas desde la Comunidad Internacional, se crea la "Oficina del Alto Representante de las Naciones Unidas para los países menos desarrollados, Estados enclavados en desarrollo y los pequeños Estados insulares en desarrollo" (UN-OHRLLS, por sus siglas en inglés) ${ }^{27}$, instando a que esta se hiciera operativa en el menor tiempo posible. Esta Oficina dependiente de la Secretaría trabaja por la defensa de este conjunto de países y da seguimiento a los proyectos de la ONU en los tres renglones en los que tiene competencias - tomando en consideración que un buen número de Estados menos desarrollados también se inserta en alguna de las otras dos categorías de Estados-. En concreto, tal y como se esboza en el punto 17b del Mecanismo de seguimiento, monitoreo y revisión en la implementación del Programa de Acción para los Países Menos Desarrollados en la década 2001-2010 28 , que de alguna manera es el instrumento guía para el funcionamiento de la UN-OHRLLS, para el caso de los pequeños Estados insulares en desarrollo, su principal cometido es brindar apoyo al BPOA de 1994.

Un aspecto que resulta de enorme interés en relación con esta Oficina es su trabajo conjunto con la Alianza de los Pequeños Estados Insulares (AOSIS, por sus siglas en inglés), que es una Organización intergubernamental creada en 1990 durante la Segunda Conferencia sobre el Clima de Ginebra. Su razón de ser es el dotar de voz a los países con mayor riesgo ante el incremento del nivel del mar por efecto del calentamiento global - por ese motivo algunos de sus 39 miembros oficiales no son islas o Estados soberanos-, y por lo tanto, se justifica perfectamente su vinculación con la UN-OHRLLS, en cuya sede tienen representación.

Además, esta Oficina trabaja en coordinación con la Red de Pequeños Estados Insulares en Desarrollo (SIDSnet) que se funda en 1997 para el intercambio de información entre sus miembros, que son los mismos que componen la AOSIS. Según su propia página web, sus cometidos son: dar seguimiento a las reuniones que incumban a los SIDS (acción que lleva a cabo conjuntamente con el Instituto Internacional para el Desarrollo Sostenible, de Canadá), completar la carencia de datos sobre el desarrollo sostenible de los SIDS y facilitar la cooperación, la acción y el apoyo en pos del desarrollo sostenible de estos Estados. Esta plataforma se encuentra totalmente financiada por la Secretaría General para la Cooperación Internacional al Desarrollo del gobierno de España dentro de un proyecto sobre "Desarrollo de Capacidades a través de la Educación para el Desarrollo Sostenible y Gestión de Conocimiento en Pequeños Estados Insulares en Desarrollo" ${ }^{29}$. Este proyecto se inserta en el marco del Consorcio Universitario de Estados Insulares, que es una interesante iniciativa que se crea en 2005 durante la Segunda Conferencia sobre SIDS de las islas Mauricio, aunque la idea surge durante la Mesa Redonda Ministerial auspiciada por el PNUD en Jamaica en mayo de $2002^{30}$.

\footnotetext{
27 "Third United Nations Conference on the Least Developed Countries", UN General Assembly, 56 ${ }^{\text {th }}$ session, resolution 56/227, art.1. A/RES/56/227 (24 de diciembre de 2001), en http://www.worldlii.org/int/other/UNGARsn/2001/304.pdf.

28 "Follow-up mechanism for coordinating, monitoring and reviewing the implementation of the Programme of Action for the Least Developed Countries for the Decade 2001-2012", UN General Assembly, 56 ${ }^{\text {th }}$ session, Third United Nations Conference on the Least Developed Countries: implementation of the Programme of Action for the Least Developed Countries for the Decade 2001-2010, A/56/645 (23 November 2001), en http://www.unohrlls.org/UserFiles/File/LDC\%20Documents/Reports/N0165665_A\%2056\%20645.pdf.

29 "El SGCID firma un acuerdo con NNUU para la educación por el desarrollo sostenible en los Estados insulares en desarrollo", Misión permanente deEspaña ante las Naciones Unidas (Mayo 2012), en http://www.spainun.org/2012/05/el-sgcid-firma-un-acuerdo-con-nnuu-para-la-educacion-por-el-desarrollosostenible-en-los-estados-insulares-en-desarrollo/.

${ }^{30}$ UNESCO: "Intersectoral Platform on Small Island Developing States", en http://portal.unesco.org/en/ev.php-URL_ID=32856\&URL_DO=DO_TOPIC\&URL_SECTION=201.html.
} 


\section{Conferencia de las Naciones Unidas sobre el Desarrollo Sostenible de 2002}

Esta tercera Cumbre de la Tierra (la primera fue en Estocolmo en 1972 y la segunda fue la de Río de Janeiro de 1992), celebrada en Johannesburgo entre el 26 de agosto y el 4 de septiembre de 2002, no tuvo la producción documental de la anterior, si bien su cometido principal fue la promoción del desarrollo sostenible y la consecución de compromisos concretos para el cumplimiento completo de la Agenda 21. El llamado Plan de Implementación de Johannesburgo ${ }^{31}$ (A/CONF.199/20), también conocido como JPOI, que separa en once capítulos las líneas de acción que considera prioritarias, dedica el capítulo séptimo a los SIDS, en el que se reconoce que estos territorios se encuentran muy condicionados por una buena cantidad de factores adversos que dificultan la completa adopción del $B P O A^{32}$. Las principales novedades recogidas en las prioridades concretas para pequeños Estados insulares en desarrollo que se identifican en el Plan de Implementación son: la aplicación del desarrollo sostenible de las pesquerías, la asistencia a los SIDS para la gestión de sus aguas jurisdiccionales, la adopción del Programa Global de Acción para la Protección del Medio Marino frente a las actividades realizadas en tierra ${ }^{33}$, y la adopción de medidas específicas a estos territorios por parte de la Organización Mundial del Comercio.

El tono de urgencia que denota el documento motiva que, al final del capítulo, se reclame a la Asamblea General para que en su $57^{\mathrm{a}}$ sesión ordinaria ${ }^{34}$ considere la creación de una Conferencia que debería, para 2004, revisar en profundidad el Barbados Plan of Action en su décimo aniversario.

\section{Reunión Internacional de las Naciones Unidas para la Revisión del Programa de Acción para el desarrollo sostenible de los pequeños Estados insulares en desarrollo de 2005}

Esta Conferencia organizada en Port Louis (Mauricio) del 10 al 14 de enero de 2005 —unos pocos meses más tarde de lo previsto-, como su nombre oficial indica, consistió fundamentalmente en la revisión del conocido BPOA tras su primera década de puesta en marcha. Lo primero que es preciso destacar es que en la Declaración de Mauricio se afirma la validez del Plan de Acción de Barbados al tiempo que llama la atención a algunos aspectos relativamente novedosos respecto a anteriores eventos, como son la importancia de la gobernanza y el trabajo en la construcción de la resiliencia ${ }^{35}$. Sin embargo, la parte documental llamada Estrategia de Mauricio para avanzar en la implementación del Programa de Acción para el desarrollo sostenible de los pequeños Estados insulares en desarrollo (o simplemente Mauritius Strategy of Implementation; MSI) resulta ser mucho más profusa en su articulado que el BPOA.

\footnotetext{
31 "UN report of the World Summit on sustainable development", A/CONF.199/20, Johannesburg, South Africa (26 de agosto - 4 de septiembre 2002), en http://www.un.org/ga/search/view_doc.asp?symbol=A/CONF.199/20. 32 Ibid., par. 58.

${ }^{33}$ United Nations Environment Programme: "Global Programme of Action for the protection of the marine environment from land-based activities" UNEP(OCA)/LBA/IG.2/7, Washington DC (23 de octubre - 3 noviember de 1995), en: http://www.gpa.unep.org/index.php/about-gpa.

34 "Further implementation of the Programme of Action for the Sustainable Development of Small Island Developing States", UN General Assembly, 57 ${ }^{\text {th }}$ session, Resolution adopted by the General, A/RES/57/262 (28 de febrero de 2001), en http://www.preventionweb.net/files/resolutions/N0255660.pdf.

35 "UN report of the International Meeting to Review the Implementation of the Programme of Action for the Sustainable Development of Small Island Developing States", Annex I. A/CONF.207/11, Port Louis, Mauritius (10-14 January 2005), en

http://www.un.org/ga/search/view_doc.asp?symbol=A/CONF.207/11.
} 
En efecto, esta Estrategia de Implementación de Mauricio rompe algunas de las 14 esferas prioritarias del documento de Barbados y las reelabora parcialmente en las 19 "áreas prioritarias" que se enumeran a continuación ${ }^{36}$ :

- Cambio climático y elevación del nivel del mar

- Desastres naturales y medioambientales

- Gestión de residuos

- Recursos marinos y costeros

- Recursos de agua dulce

- Recursos terrestres

- Recursos energéticos

- Recursos turísticos

- Recursos de la biodiversidad

- Transportes y comunicaciones

- Ciencia y tecnología

- Graduación desde el status de "menos desarrollo"

- Comercio: liberalización y globalización

- Capacitación y educación para el desarrollo sostenible

- Producción sostenible y consumo

- Medioambiente nacional y regional

- Salud

- Gestión del conocimiento y la información para la toma de decisiones

- Cultura

La cantidad de asuntos listados señala de alguna manera la complejidad que el tratamiento de los SIDS requiere, pues tanto sus vulnerabilidades como los riesgos a los que se ven sometidos estos espacios tienen múltiples causas y efectos interrelacionados. Aunque algunos de los 19 puntos del MSI no son tan novedosos como parecieran, ya que de alguna manera buena parte de ellos se mencionan en anteriores documentos - y fortalecerían aspectos específicos de los Objetivos del Milenio-, resulta especialmente necesaria la alusión a la cuestión de la graduación desde el status de "menos desarrollo". En el apartado correspondiente, debido a que existen criterios acerca de las condiciones que constituyen un país menos desarrollado, se pide un mecanismo para que la transición de salida se realice suavemente $^{37}$. Al respecto, merece la pena comentar que hasta la fecha actual, han abandonado el grupo de países "menos desarrollados": Botswana (en 1994), Cabo Verde (en 2007), Maldivas (en 2011) y Samoa (en 2014) ${ }^{38}$.

\footnotetext{
${ }^{36} \mathrm{Ibid}$., I, anexo II, 16-82.

${ }^{37}$ Ibid., I, anexo II, 64.

${ }^{38}$ UN Department of Economic and Social Affairs, "LDC Information: Graduation and Transition Process", en: http://www.un.org/en/development/desa/policy/cdp/ldc/ldc_graduated.shtml
} 
Un último capítulo del $M S I$, tal cual sucediera con el BPOA, se ocupa de la implementación, en el cual trata aspectos como el acceso a la financiación, la transferencia de tecnología, la capacitación, la gobernanza nacional e internacional, el monitoreo y el papel de la ONU y los propios SIDS ${ }^{39}$. Además, es preciso comentar que de esta Segunda Conferencia sobre SIDS nace el Consorcio Universitario de Estados Insulares, que desde el ámbito universitario tiene encomendado el objetivo de mejorar las instituciones académicas de los Estados insulares pequeños por medio de la capacitación encaminada a la puesta en marcha del BPOA. A día de hoy el Consorcio está compuesto por cinco universidades: la Universidad de Malta, la Universidad de Mauricio, la Universidad del Pacífico Sur, la Universidad de las Islas Vírgenes y la Universidad de las Indias Occidentales. Asimismo, las Universidades de Seychelles y de las Palmas de Gran Canaria (España) están asociadas a esta entidad con un proyecto para establecer una plataforma de tecnologías de la información común a esta red interuniversitaria ${ }^{40}$.

Finalmente es preciso atribuir al MSI el nacimiento del Global Island Partnership (GLISPA) a petición de los presidentes de Seychelles y Palaos, quienes buscaban un ámbito de cooperación para compartir soluciones a problemas comunes en los ámbitos insulares. Esta iniciativa está conformada por un buen número de Organizaciones Internacionales y regionales, y además cuenta con unos 40 miembros estatales (islas pequeñas y grandes, países continentales con islas y dependencias) ${ }^{41}$. Fue creada durante la octava reunión de la Conferencia de las Partes del Convenio sobre la Diversidad Biológica de $2006^{42}$ para facilitar el diálogo entre islas para llevar a cabo acciones de conservación de la biodiversidad insular y para permitir el uso sostenible de los recursos.

\section{Reunión de Alto Nivel para la revisión de la Estrategia de Implementación de Mauricio para avanzar en la implementación del Programa de Acción para el desarrollo sostenible de los pequeños Estados insulares en desarrollo, en 2010}

Tal cual sucediera en 1997, cuando la Asamblea General considera necesaria la revisión del $B P O A$ que se llevaría a cabo dos años más tarde, el principal órgano deliberativo de la ONU emite una resolución (A/RES/62/191) en diciembre de 2007 en la que insta a la revisión de la Estrategia de Implementación de Mauricio ${ }^{43}$. En posteriores resoluciones ${ }^{44}{ }^{45}$ se desarrollaría la estructura del evento que tendría lugar el 24 y 25 de septiembre de 2010, que en esta

\footnotetext{
${ }^{39}$ A/CONF.207/11, I, anexo II, 83-104.

${ }^{40}$ UNESCO... Op., Cit.

${ }^{41}$ UNDP. SIDS-SIDS success stories: An innovative partnership in South-South cooperation. 2010. [en línea]. [Consulta: 19 de diciembre 2014]. Disponible en:

http://www.sidsnet.org/sites/default/files/sids-sids_success_stories.pdf.

${ }_{42}$ Convention on Biological Diversity: "Conference of the Parties", en http://www.cbd.int/cop/default.shtml.

43 "Follow-up to and implementation of the Mauritius Strategy for the Further Implementation of the Programme of Action for the Sustainable Development of Small Island Developing States" UN General Assembly, 62 ${ }^{\text {th }}$ session, Resolution adopted by the General Assembly on 19 December 2007, A/RES/62/191, en http://www.un.org/en/ga/search/view_doc.asp?symbol=A/RES/62/191.

44 "Follow-up to and implementation of the Mauritius Strategy for the Further Implementation of the Programme of Action for the Sustainable Development of Small Island Developing States UN General Assembly", 63 ${ }^{\text {th }}$ session, Resolution adopted by the General Assembly on 19 December 2008. A/RES/63/213, en http://www.un.org/en/ga/search/view_doc.asp?symbol=A/RES/63/213.

45 "Follow-up to and implementation of the Mauritius Strategy for the Further Implementation of the Programme of Action for the Sustainable Development of Small Island Developing States", UN General Assembly, 64 ${ }^{\text {th }}$ session, Resolution adopted by the General Assembly on 21 December 2009, A/RES/64/199, en http://www.un.org/en/ga/search/view_doc.asp?symbol=A/RES/64/199.
} 
ocasión estaría conformado por paneles preparatorios que trabajarían desde febrero hasta mayo por áreas geográficas. Durante los preparativos el Departamento de Asuntos Económicos y Sociales de la ONU preparó un informe que serviría de apoyo, titulado Trends in sustainable development: Small Island Developing States (SIDS) $2010^{46}$, mientras que de las diferentes reuniones por regiones se obtuvieron los insumos que completarían el reporte final.

De la reunión propiamente dicha, también llamada "MSI+5", se extraen dos documentos; uno declarativo ${ }^{47}$ y otro de naturaleza evaluativa ${ }^{48}$. En el primero, que es de mayor tamaño que los de años anteriores, se vuelve a llamar la atención sobre las necesidades especiales de los SIDS y sus progresos, aunque se recalca que estos son relativos por la falta de medios de estos países, por sus conocidas vulnerabilidades y por su escasa incidencia en los avances hacia la consecución de los Objetivos del Milenio. Además, en virtud de la resolución 63/281 de 2009 se introduce la mención expresa a la cuestión de seguridad que deriva del cambio climático ${ }^{49}$, llamando la atención acerca de la necesidad de contar con herramientas de gestión de riesgos y de prevención de desastres naturales ${ }^{50}$, de acuerdo con los lineamientos del Hyogo Framework of Action 2005-2015 ${ }^{51}$, que es el documento aprobado internacionalmente en esta materia durante la Conferencia Internacional para la Reducción de Desastres de 2005.

El segundo documento, la revisión [de los cinco años] de la Estrategia de Implementación de Mauricio para avanzar en la implementación del Programa de Acción para el desarrollo sostenible de los pequeños Estados insulares en desarrollo, tiene dos partes principales; una en relación con los progresos realizados por los SIDS (en renglones macroeconómicos y en relación con los Objetivos del Milenio), y otro sobre lecciones aprendidas y retos. Acerca de esta primera parte, además llama la atención que se ponga de manifiesto de manera expresa la vulnerabilidad de los SIDS ante "los shocks", o crisis internacionales de alcance planetario, entre los que cita "la crisis financiera de 2007-2010 y la crisis de alimentos y combustible de 2007-2008 [...]" ${ }^{\prime 52}$. En cuanto a las lecciones aprendidas y retos en la puesta en funcionamiento de la MSI se repasan las formas de implementación del texto de 2005 que ya se mencionaron en el anterior apartado ${ }^{53}$. La exhaustividad analítica de esta sección es llamativa, pues desglosan pormenorizadamente, y con ejemplos cuantitativos, diferentes aspectos de interés de cada uno de las 19 áreas prioritarias que se consideraron

\footnotetext{
46 "Trends in sustainable development: Small Island Developing States (SIDS) 2010", UN Department of Economic and Social Affairs, New York, United Nations, en http://www.sidsnet.org/sites/default/files/resources/trends_in_sustainable_development_sids.pdf.

47 "Outcome document of the High-level Review Meeting on the Implementation of the Mauritius Strategy for the Further Implementation of the Programme of Action for the Sustainable Development of Small Island Developing States", UN General Assembly, 65 ${ }^{\text {th }}$ session, resolution adopted on 25 September 2010, A/RES/65/2, en

http://www.un.org/en/ga/search/view_doc.asp?symbol=A/RES/65/2.

48 "Follow-up to and implementation of the Mauritius Strategy for the Further Implementation of the Programme of Action for the Sustainable Development of Small Island Developing States", UN General Assembly, 65 ${ }^{\text {th }}$ session, Sustainable development:. Five-year review of the Mauritius Strategy for the Further Implementation of the Programme of Action for the Sustainable Development of Small Island Developing States, A/65/115, en http://www.un.org/en/ga/search/view doc.asp?symbol=A/65/115.

${ }^{49}$ UN General Assembly, 63 ${ }^{\text {rd }}$ session, resolution adopted on 3 June 2009, Climate change and its possible security implications. A/RES/63/281, en http://www.un.org/es/comun/docs/?symbol=A/RES/63/281.

${ }^{50} \mathrm{~A} / \mathrm{RES} / 65 / 2$, par. 12.

${ }^{51}$ UN report of the World Conference on Disaster Reduction, Hyogo Declaration, Kobe, Hyogo, Japan (18-22 de enero de 2005), A/CONF.206/6, en http://www.un-documents.net/hyogodec.htm.

${ }_{53}$ A/65/115, par. 12.

${ }^{53}$ A/CONF.207/11, I, anexo II, 83-104.
} 
para la $M S I^{54}$. Finalmente, se mencionan algunos aspectos que deben tenerse en cuenta en el futuro, como son: el fortalecimiento del apoyo hacia el planeamiento nacional (especialmente para fomentar la resiliencia), la creación de perfiles para SIDS de vulnerabilidad/resiliencia, mayor concentración en las áreas temáticas clave (sobre todo para priorizar, ya que no suele haber recursos para atender todas), apoyo a las iniciativas de colaboración (regional, internacional, con ONG, con Organizaciones Internacionales,...) para la entrada en funcionamiento completa del BPOA, el trabajo en el acceso a los recursos financieros, y la institucionalización de la ayuda especial a $\operatorname{los} \operatorname{SIDS}^{55}$.

\section{Conferencia de las Naciones Unidas sobre el Desarrollo Sostenible de 2012}

Esta Conferencia Internacional, también publicitada como "Cumbre de Río +20", se centró en dos bloques temáticos: "economía verde en el contexto del desarrollo sostenible y la erradicación de la pobreza" y "el marco institucional para el desarrollo sostenible". Especialmente interesante resulta el hecho de que, con anterioridad a la fecha de celebración del evento que volvería a editarse en Río de Janeiro entre el 20 y el 22 de junio de 2012, los SIDS, tal cual hiciera para el MSI+5, se llevaron a cabo reuniones de trabajo por bloques geográficos con motivos preparatorios. Igualmente es necesario mencionar que durante los días que duró la Cumbre se celebraron decenas de "side events", o actividades temáticas sobre un buen número de asuntos medioambientales relacionados con las reuniones principales, aunque celebrándose al mismo tiempo. Nueve de estas actividades, algunas de ellas organizadas por delegaciones de gobiernos (destaca la organizada por Maldivas para contar su experiencia), entidades como la Commonwealth, o instituciones especializadas, versaban sobre temas que involucraban de alguna manera a los pequeños Estados insulares en desarrollo.

El documento principal de esta Cumbre se titula "el futuro que queremos", y lo recoge la resolución A/RES/66/288 ${ }^{56}$. Se trata de un extenso articulado de hasta 283 puntos en el que se abordan una enorme cantidad de aspectos centrales a la temática medioambiental, aunque también otros transversales. Sobre los SIDS hay un pequeño capítulo de tres artículos en los que vuelve a transmitir la preocupación por la vulnerabilidad de estos espacios al tiempo que se incluye la alusión a los Objetivos del Milenio no cumplidos, se reitera la validez del BPOA y se insta a la celebración de la que sería la tercera — y última, hasta la fecha actualConferencia exclusivamente convocada para tratar los asuntos que preocupan a los pequeños Estados insulares en desarrollo, ya en $2014^{57}$. En cualquier caso, es preciso aclarar que a lo largo del texto, sobre todo en los capítulos que versan sobre los océanos o la reducción de riesgos, se alude con frecuencia y de manera expresa a la situación de los SIDS.

\section{Tercera Conferencia de las Naciones Unidas sobre los Pequeños Estados Insulares en Desarrollo, de 2014}

Esta Conferencia, celebrada en Apia (Samoa) del 1 al 4 de septiembre de 2014, es hasta la fecha el último gran evento mundial focalizado en el caso y las necesidades concretas de los SIDS. Destacó especialmente por una elevada participación de Estados, medios de

\footnotetext{
${ }_{55}^{54} \mathrm{~A} / 65 / 115$, par. 23-117.

${ }^{55}$ Ibid., par. 118-127.

56 "The future we want", UN General Assembly, 66 ${ }^{\text {th }}$ session, resolution adopted on 27 July 2012 , A/RES/66/288, en http://www.un.org/ga/search/view_doc.asp?symbol=A/RES/66/288.

${ }^{57}$ Ibid., par. 178-80.
} 
comunicación y sociedad civil (ONG, sector privado...), que se manifestó en un gran número de actividades paralelas a las reuniones plenarias del Congreso, tanto con anterioridad como durante el mismo, entre las que hay que mencionar los "diálogos multipartitos de cooperación" acerca de materias variadas como el desarrollo económico sostenible o los asuntos relacionados con la salud y el bienestar de mujeres y jóvenes. La modalidad de trabajo por paneles geográficos fue similar a la iniciada en el MSI+5 del 2010, pues se contó con los insumos aportados en 2013 por las regiones de trabajo del UN-OHRLLS: Caribe, Pacífico y AIMS (África, Índico, Mediterráneo y Sur de China) y por los reportes nacionales aportados por un buen número de Estados insulares.

El producto más importante del evento es el documento Small Islands Developing States Accelerated Modalities of Action [modalidades aceleradas de acción para los SIDS], anunciado y publicitado como el SAMOA Pathway, en clara alusión a la ya comentada "graduación desde el estatus de menos desarrollo" del país anfitrión este mismo año, y adoptado por la Asamblea General el 14 de noviembre de este mismo año ${ }^{58}$. En el preámbulo del SAMOA Pathway ${ }^{59}$ se vuelve a reiterar la necesidad de seguir trabajando en los Objetivos del Milenio y en la consecución total del BPOA y el MSI, pues se advierte de que, pese a los avances, algunos retos persisten, sobre todo debido a las condiciones geográficas de estos territorios insulares de escaso tamaño y la posición que habitualmente estos ocupan en el planeta. Es en este contexto en el que se explica que el enfoque de trabajo de este documento se centre en los asuntos prioritarios que se identificaron y sobre los que se establecen líneas claras de acción para cada una de ellas. Estas 15 prioridades acordadas son, por orden de aparición en el texto original ${ }^{60}$ :

- Crecimiento económico sostenible y sustentable, inclusivo y equitativo con trabajo decente (incluye dos bloques de medidas: desarrollo de modelos en los SIDS para la implementación del desarrollo sostenible y la erradicación de la pobreza y turismo sostenible)

- Cambio climático

- Energía sostenible

- $\quad$ Reducción de riesgos ante desastres

- Océanos y mares

- Seguridad alimentaria y nutrición

- Agua y saneamientos

- Transporte sostenible

- $\quad$ Producción y consumo sostenible

- Gestión de químicos y residuos, incluyendo aquellos que son peligrosos

- Sanidad y enfermedades no comunicables [a la OMS: aquellas no infecciosas y no transmisibles]

- $\quad$ Igualdad de género y empoderamiento de la mujer

\footnotetext{
58 "Small Island Developing States Accelerated Modalities of Action (Samoa Pathway)", Annex, UN Report on the Third International Conference on Small Islands Developing States, Apia, Samoa, (1-4 de septiembre 2014), A/CONF.223/3, en http://www.sids2014.org/content/documents/358A-CONF-223-5\%20ENGLISH.pdf.

${ }^{59}$ Ibid..

${ }^{60}$ Ibid., arts. 23-95.
} 
- Desarrollo social (incluye dos bloques de medidas: promoción de sociedades pacíficas y comunidades seguras, y educación)

- Biodiversidad (incluye dos bloques de medidas: desertificación y degradación del suelo, y bosques)

- Especies invasivas no autóctonas

Estas 15 áreas prioritarias (18, si se separan los puntos con dos bloques de medidas) vuelven a rehacer la lista de los dos documentos anteriores, aunque en buena medida todos sus puntos siguen estando presentes de alguna manera. No obstante, en la línea del $M S I+5$, que recomendaba priorizar, es evidente que en el SAMOA Pathway se denota un esfuerzo por ser bastante específico en aquellos aspectos concretos que se consideran vitales en su pronta resolución. En cualquier caso, en el último apartado del texto acerca de las vías de implementación, se vuelven a destacar los aspectos habitualmente considerados en anteriores eventos (cooperación, financiamiento, comercio, capacitación, tecnología, y datos y estadísticas para el seguimiento), además de uno nuevo relativo al apoyo institucional de la ONU a los SIDS que solicita al Secretario General la revisión del propio sistema de apoyo de esta Organización a estos territorios para que los hallazgos sean tratados en la Asamblea General en su $70^{\circ}$ período de sesiones para $2015^{61}$.

\section{Conclusiones}

En diciembre de 2012 la Asamblea General de la ONU adoptó una resolución para nombrar al 2014 como el año internacional de los pequeños Estados insulares en desarrollo ${ }^{62}$. La consecución de este hito, coincidente en el mismo año que la Tercera Conferencia Internacional sobre SIDS celebrada en Samoa, fue posible por la institucionalización de la causa de este grupo de países desde la coyuntura presentada debido a la conciencia medioambiental que despertaba en los '90 en la arena internacional. Si bien es cierto que los Estados insulares supieron posicionarse en común en torno a su preocupación al respecto del cambio climático y la elevación del nivel del mar, hay que destacar que cada Conferencia Internacional se ha caracterizado por la progresiva integración de otros muchos elementos en el análisis, sobre todo la última. Esta necesaria multidisciplinariedad que se requiere para abordar el estudio de las islas pequeñas pasa por integrar un gran número de elementos que se reflejaron en los documentos emanados en cada una de las tres ediciones de las Conferencias SIDS y de las dos revisiones quinquenales a las de 1994 y 2005. Las Cumbres de la Tierra y la creación de la UN-OHRLLS (con sus vínculos con otras entidades fuera del espectro de la ONU) completan los momentos clave tomados en cuenta para este ensayo.

Cada documento analizado tiene un sello propio, pero que no puede entenderse sin el contexto de otros eventos que se iban produciendo en los años anteriores. Así, el BPOA de 1994 es deudor indiscutible de la Agenda 21 que había sido aprobada dos años antes y cuyas referencias aún resuenan en las resoluciones de la Asamblea General. Acaso por este motivo se comprende que siete de sus 14 esferas prioritarias tengan bastante que ver con cuestiones relacionadas con el medioambiente. Esta circunstancia ya cambia para el MSI de 2005, pues, aunque siguen manteniéndose los mencionados siete puntos prioritarios, en este documento se

\footnotetext{
${ }^{61}$ Ibid., art. 119.

62 "International Year of Small Island Developing States UN General Assembly", $67^{\text {th }}$ session, resolution adopted on 21 December 2012, A/RES/67/206, en

http://www.un.org/en/ga/search/view_doc.asp?symbol=A/RES/67/206.
} 
lleva a cabo una reelaboración de los anteriores y se añaden cinco más, dotando de mayor importancia a aspectos económico-comerciales y culturales. En 2014, por el contrario, los puntos se reducen a 15 pero engloban áreas de actuación muy específicas que suelen integrar la combinación entre lo natural y lo antrópico. Esta última refocalización no refleja otra cosa que la necesidad de priorizar, ya que a lo largo de las dos décadas analizadas, cada documento repetía la necesidad de avanzar en las materias que preocupan a los SIDS, aunque siempre recordando la vulnerabilidad y escasos recursos con los que cuentan estos Estados para hacer frente a los retos y contingencias a las que deben enfrentarse.

De cualquier modo, el recorrido a través de los principales hitos para los intereses del conjunto de los SIDS permite comprobar que la Comunidad Internacional está cada vez más involucrada en su progreso, pues en un mundo global e interdependiente, los asuntos que benefician a este grupo de países son a su vez positivos para el resto del mundo. En efecto, la focalización en los SIDS lleva implícito un trabajo transversal en materias que trascienden las islas y que están desde hace tiempo en la palestra. Así, pese a que resta aún mucho por avanzar — como se reitera sin excepción desde los '90-, existen motivos para el optimismo, pues son precisamente de estos espacios insulares, remotos y sin recursos desde donde llegan las últimas noticias exitosas de "graduaciones desde estatus de menos desarrollo". El hecho de que tres de los —únicos- cuatro Estados que ya no son considerados como menos desarrollados por la ONU sean islas (Cabo Verde, Maldivas y Samoa) es muy revelador, sobre todo si se tiene en cuenta que, en la lista de posibles candidatos hay tres miembros de AOSIS: Guinea Ecuatorial, Vanuatu, Kiribati y Angola ${ }^{63}$.

63 "The Least Developed Countries report 2014. Growth with structural transformation: A post-2015 development agenda", United Nations Conference on Trade and Commerce (2014), UNCTAD/LDC72014., p III, en http://unctad.org/en/PublicationsLibrary/ldc2014_en.pdf. 